\title{
Consumer preferences for teledermoscopy screening to detect melanoma early
}

\author{
Authors: Jean Spinks ${ }^{1}$, Monika Janda ${ }^{2}$, H. Peter Soyer ${ }^{3}$, Jennifer A. Whitty ${ }^{4}$
}

\section{Affiliations:}

1. Menzies Health Institute Queensland, Griffith University

2. School of Public Health and Social Work, Institute of Health and Biomedical Innovation Queensland University of Technology, Brisbane, Queensland, Australia

3. Dermatology Research Centre, The University of Queensland, School of Medicine, Translational Research Institute, Brisbane, Australia

4. School of Pharmacy, The University of Queensland

Acknowledgements: This research was conducted with the support of the Centre of Research Excellence in Telehealth funded by NHMRC (grant ID: APP1061183) and an internal grant from Menzies Health Institute Queensland, Griffith University. The authors would like to acknowledge the contribution of Professor David Whiteman to this project as well as Mr Riyaz Shakya for his early assistance with survey development.

Conflict of interest: H.P.S. is a shareholder of e-derm consult GmbH and MoleMap by Dermatologists Ltd Pty. He provides teledermatological reports regularly for both companies. M.J. was funded by a National Health and Medical Research Council (NHMRC) career development award (No. 1045247). J.S. \& J.W have nothing to declare. 


\begin{abstract}
Introduction: 'Store and forward' teledermoscopy is a technology with potential advantages for melanoma screening. Any large-scale implementation of this technology is dependent on consumer acceptance.

Aim: To investigate preferences for melanoma screening options compared to skin selfexamination in adults considered to be at increased risk of developing skin cancer.

Methods: A discrete choice experiment (DCE) was completed by 35 consumers, all of whom had prior experience with the use of teledermoscopy, in Queensland, Australia. Participants made 12 choices between screening alternatives described by seven attributes including monetary cost. A mixed logit model was used to estimate the relative weights that consumers place on different aspects of screening, along with the marginal willingness to pay for teledermoscopy as opposed to screening at a clinic.

Results: Overall, participants preferred screening/diagnosis by a health professional rather than skin self-examination. Key drivers of screening choice were for results to be reviewed by a dermatologist; a higher detection rate; fewer non-cancerous moles being removed in relation for every skin cancer detected; and less time spent away from usual activities. On average, participants were willing to pay AU $\$ 110$ to have teledermoscopy with dermatologist review available to them as a screening option.
\end{abstract}

Discussion \& Conclusions: Consumers preferentially value aspects of care that are more feasible with a teledermoscopy screening model, as compared to other skin cancer screening and diagnosis options. This study adds to previous literature in the area which has relied on the use of consumer satisfaction scales to assess the acceptability of teledermoscopy. 


\section{Introduction}

The state of Queensland in Australia has the highest rate of skin cancer in the world including the highest rate for melanoma mortality [1]. In 2011, there were 3,249 new cases of melanoma diagnosed in Queensland, which was the second leading form of cancer for both men and women [2]. In addition, around 133,000 cases of non-melanoma skin cancer are diagnosed in the state each year [3]. This presents a major challenge to health services in terms of the high number of cases, their differential diagnosis and often late presentation of tumours. The three main types of skin cancer detected are basal cell carcinoma (BCC), squamous cell carcinoma (SCC) and melanoma [4]. It is important to find all melanomas very early as recent results have shown that even those under $1 \mathrm{~mm}$ thick may lead to death [5]. BCCs and SCCs also benefit from earlier detection, but due to their lower mortality rates, less crucially so. Despite this there is currently no population-based screening program implemented in Queensland or Australia. People are advised to check their own skin (skin self-examination SSE) and present to a doctor urgently if any spots or moles change $[6,7]$. Opportunistic screening is also performed by General Practitioners (GPs)(family doctors) and increasingly by skin cancer clinics, which are generally staffed by GP's, some with additional training [8]. Dermatologists cannot be accessed directly by consumers in Australia, requiring a referral from a GP.

Teledermoscopy is a technology that captures images of potential skin cancers using a hand-held dermatoscope. 'Store and forward' technology is used to upload and send the image for diagnosis $[9,10]$. This technology is now available as a hardware addition to a mobile phone and consumers can submit good quality images for triage with minimal training [11]. Studies have found that results from teledermoscopy images, reviewed by an experienced teledermatologist, have high concordance with face-to-face diagnoses, as well as approximating $100 \%$ sensitivity and around $90 \%$ specificity to differentially diagnose melanoma and non-melanoma skin cancers [11-14]. Thus, teledermoscopy has the potential to assist with managing clinician workloads, increasing access to dermatologists and potentially avoiding unnecessary biopsies and other investigations which occur at a higher rate when performed by non-dermatologist and less experienced clinicians [15].

However consumer acceptance of this approach has yet to be established and is essential if broad implementation of this strategy is to be considered. Consumer preferences have predominantly been measured by 'consumer satisfaction' rating scales, with somewhat mixed results [16-22]. A review paper by Demiris et al. (2004) identified 14 studies related to consumer satisfaction and acceptance of teledermatology applications more broadly (including both the 'store and forward' technology as well as video conferencing). The authors concluded that the concept of satisfaction is multidimensional and should cover many underlying factors including convenience of the service; confidence in the result; and ease of communication with the clinician [18]. However, one of the main limitations with using satisfaction rating scales is that they are unidimensional.

A Discrete Choice Experiment (DCE) is a type of survey which elicits consumer preferences around service delivery in a number of different disciplines, including increasingly in health [2325]. DCEs are designed to simulate real-life consumer choice situations where more or less attractive characteristics of a product or service are traded relative to alternative options. In this 
way, the key average drivers of choice for a population can be determined and the potential value uptake of a new service estimated. In this study, we use DCE methodology to measure consumer preferences around the key characteristics of skin cancer screening options including teledermoscopy, in order to provide insights into their acceptability. In this study, we investigate the preferences for skin cancer screening options compared to skin self-examination in a group of people considered to be at increased risk of developing skin cancer.

\section{Methods}

A survey containing a DCE was developed and administered according to best practice guidelines [26]. Participants were asked to make 12 choices between two 'generic' health service options describing different combinations of screening services, either currently available or possible in the near future. Each screening alternative was described according to seven different screening attributes, the levels of which varied across the different alternatives in each choice set (Table 1).

\section{$<\quad$ Table 1 about here $\quad>>$}

These alternatives, and the different attribute levels over which they vary, were chosen based on the results of three previous surveys undertaken in the target group [11], as well as a literature review $[14,16-22,27,28]$ and included important screening outcome, financial and convenience considerations. In each choice set participants were also asked to compare these service options with two 'opt out' options - either skin self-examination (that is, performing a skin check on your own without the help of a health professional), or not to undertake any screening at all.

The screening alternatives described by the attributes and levels in Table 1 were allocated into choice sets using a multinomial logit (mnl) d-efficient design, assuming no prior information about the likely magnitude or direction of coefficients (zero priors) [29, 30]. This optimised the likelihood of the design estimating statistically efficient preference parameters, despite the relatively small sample size and inclusion of opt out alternatives. To avoid unrealistic combinations of attributes and levels, a constraint was added to the design, since consumers cannot access a dermatologist for screening without a GP referral in Australia. Specifically, review by a GP was constrained to only appear with screening being performed at a skin cancer or GP clinic; whereas, screening by teledermoscopy could be reviewed by either a GP or a dermatologist. To reduce participant burden, two versions of the survey were created with 12 unique choice questions contained in each version; participants were randomly allocated to each version. An example choice set is presented in Table 2.

$$
<\quad \text { Table } 2 \text { about here } \quad>>
$$

The survey also collected information on socio-demographic characteristics; current and intended skin-screening practices; and the level of concern about developing skin cancer in the future. These questions had been validated before collection in previous questionnaires; however the choice questions had not been tested previously. 


\section{Participant recruitment}

As prior experience of a good or service can be a strong influence on choice [31], it was decided to control for this by recruiting a sample who all had previous experience with teledermoscopy in a pilot teledermoscopy trial [11]. Participants from this trial who agreed to further contact were invited via email to complete the survey either online or on a paper-pencil copy we mailed to them. The inclusion criteria for the original study included: age 50-64 years; living in Queensland (residing in Brisbane or willing to travel); and considered to be at moderate or high risk for melanoma (they had to meet one of the following; fair eye, hair or skin type, previous skin excisions, or a personal or family history of melanoma). There were no exclusion criteria [11].

\section{Analysis of the choice data}

The DCE data were analysed using non-linear regression models, in which the attribute levels (independent variables) were used to explain participant screening choice (dependent variable). Here, we use a mixed-logit model (MMNL) which offers advantages to the more often used multinominal logit (MNL) [32, 33]. Details of the theoretical framework are provided in Appendix 1. A number of assumptions were made. Firstly, as no participants chose the "I would prefer not to undertake any screening" opt out option, this alternative was not included in the model. Instead, skin-self-examination was assumed to be the base alternative, the utility of which was assumed to be constant. All attributes except cost were effects coded to allow for non-linear relationships, especially for qualitative (unordered) variables. Costs were coded continuously. All attributes were initially modelled as random parameters. All distributions around random parameters were assumed to be normal and estimated using 1000 halton draws (this is a quasirandom or "intelligent" method for simulating distributions often used in choice modelling [33]). If the standard deviations of the random parameters were found to be non-significant at the $10 \%$ level, the attribute was modelled as a non-random parameter. Similar to linear regression, a positive coefficient means that the attribute level of interest is preferred over those with a negative coefficient.

\section{Willingness to pay estimates}

Using the outputs from the model derived above, an indicative marginal willingness to pay (MWTP) was estimated for the hypothetical policy change from a situation where only skin-selfassessment, GP screening and skin cancer clinic screening is available, to a situation where the option of teledermoscopy with review by a dermatologist is additionally available. This provides an estimate of the monetary value for the welfare gain (or loss) for consumers by having teledermoscopy available to them as an alternative. Following Lanscar [34] and Ryan [35], we use the method for compensating variation described by Small and Rosen [36] to estimate the welfare gain associated with different screening models. Compensating variation is a measure of how much money needs to be given or taken from a consumer after a price or quality change to leave them with their initial level of satisfaction (known as utility) [34]. This method accounts for both the relative importance of a given attribute level(s) as well as the probability of choosing an alternative which contains that particular attribute (Table 4). The Small and Rosen formula used is detailed in Appendix 2. 


\section{Results}

Completed DCE surveys were returned by 35 participants (70\% participation rate). There were no partial completions. A summary of the characteristics of the participants who completed the survey is presented in Table 3 .

$$
<\quad \text { Table } 3 \text { about here } \quad>>
$$

As can be seen from Table 3, participants were between 50 and 64 years, representing the age group with the highest incidence of melanoma (this was an inclusion criteria of the original trial). On average the participants had high levels of education and income and were predominantly residing in major cities.

There were no missing choice data. Approximately half of the sample completed each survey version (version 1 16/35 or 46\%; version 2 19/35 or 54\%). No respondents opted for the alternative 'no screening', indicating that early detection of melanoma was of high relevance to all participants. The 'no screening' option was therefore removed from the models. The skin selfassessment alternative was chosen on 36 separate occasions (choice sets) by 6 different participants. This is a small proportion $(36 / 420 ; 8.6 \%)$ of the total possible 420 choice sets across all 35 participants, suggesting a strong preference in this cohort to take up a form of screening that involves a medical doctor.

Results from the MMNL model are presented in Table 4.

$$
\quad<\quad \text { Table } 4 \text { about here } \quad>>
$$

Participants strongly preferred their screening results to be reviewed by a dermatologist rather than a GP; a high chance ( $>95 \%$ ) of detection during screening; that less non-cancerous moles (3) are removed to detect one skin cancer, rather than more (10); and lower cost (all pvalues $<0.05)$. Not spending greater than 4 hours away from usual activities was preferred, but this was only significant at the $10 \%$ level. No significant difference was found between the different screening methods in terms of their effect on screening choice (teledermoscopy compared to visiting a skin cancer clinic or a GP). However, teledermoscopy results were the only results reviewed by a dermatologist in the available choices and participants had been made aware of this in the survey. There was a non-linear preference observed for the levels of the attribute "length of time to receive results", with a wait time of up to one day preferred to less than 4 hours; however, this relationship was only significant at the $10 \%$ level. Participating in a screening service involving a doctors' opinion either during a face-to-face visit or by telediagnosis was strongly preferred over skin self-examination as shown by the negative and statistically significant constant for this alternative. We tested a range of additional variables to investigate whether they explained the variation in participant choices for screening. These included the participant socio-demographics shown in Table 3, whether participants had previously been diagnosed with skin cancer, if they had a skin cancer detected during the trial, if they were more worried about developing skin cancer in the future and if they currently performed skin self-examination. None of these variables were shown to significantly explain heterogeneity around choices and they were therefore excluded from the final model. 
The indicative marginal willingness-to-pay (MWTP) calculation is shown in Table 5.

$$
<\quad \text { Table } 5 \text { about here } \quad>>
$$

Here, we consider an initial 'state of the world' as being comprised of three different screening options, which were chosen as being most indicative of the current options available: skin selfexamination, skin cancer clinic or GP screening. We assume a 1-2 hour distraction from usual activities, greater than $95 \%$ chance of detecting a skin cancer if one is present, a wait time of less than 4 hours for results, that the results are reviewed by a GP, and that 5 non-cancerous moles are removed for every skin cancer detected for services provided in a skin cancer or GP clinic. The introduction of a teledermoscopy alternative as an additional (fourth) screening mode, assumes review by a dermatologist and that only 3 non-cancerous moles need to be removed to detect one skin cancer. The use of a lower rate of non-cancerous mole removal by dermatologists has been justified by previous findings [15]. This change, from three screening options to four screening options is associated with an average welfare improvement of AU $\$ 110$ (estimated using the Small \& Rosen compensating variation equation (Appendix 2). This is interpreted as consumers being willing to pay an average of $\$ 110$ to move the current situation where they can choose between skin self-examination, skin cancer clinic and GP screening alternatives only, to a situation where all of these options plus teledermoscopy with dermatologist review being available. The likelihood of uptake of the different alternatives also changes (Table 5). Whilst the skin cancer clinic model is the most demanded initially $(0.548$ probability of uptake), the new teledermoscopy model is the most likely to be taken up after its introduction (probability of uptake 0.668). This likelihood of uptake and welfare gain is driven primarily by having review of the results by a dermatologist rather than a GP.

Given the strong preference for skin examination by a dermatologist, we conducted further hypothetical modelling assuming that dermatologists would staff skin cancer clinics and achieve a lower rate of mole removal ( 3 for every skin cancers detected). In this scenario, the welfare improvement associated with the teledermoscopy (fourth) option is estimated to be AU\$52 (results not presented). Thus, teledermoscopy still provides additional welfare gains to consumers beyond access to a dermatologist and a lower rate of mole removal.

\section{Discussion}

This study uses discrete choice methods to elicit consumer preferences for skin cancer screening services, including novel teledermoscopy screening. This new technology offers consumers the chance to 'store and send' an image of any potential skin cancer for review by a health professional. The findings suggest that people 50-64 years at high risk of skin cancer strongly preferred their results to be reviewed by a dermatologist - an option that was only available using teledermoscopy in the survey, and would likely only be available via teldermoscopy for the majority of consumers in the Australian health system, where family doctors must be consulted first before any specialist appointment. Results also show that a higher skin cancer detection rate and lower rate of removal of non-cancerous lesions are also strongly preferred, as is a shorter time away from usual activities. There was an unexpected non-linear preference observed for levels of the attribute length of time to receive results. However, this was not significant at the 
conventional 5\% level. Furthermore, any such trend could indicate that people prefer a fast, but also thorough assessment of their skin lesions.

Many of the results found supporting the importance of particular attributes in this study are in concordance with the previous literature focusing on consumer satisfaction with teledermoscopy or teledermatology more broadly $[14,18,19,27,28]$. For example, Whited et al. found that the majority of consumers agreed that they had confidence that dermatologists can diagnose teledermatology pictures and that a teledermatology consult is more convenient than going to a dermatologist clinic [22]. Qualitative and quantitative results from Collins et al. show no difference in the overall satisfaction depending on the type of consultation (face to face compared with teledermatology) [17] and that both groups were happy with their care. This is despite concerns by some consumers that they would like more personal or face-to-face communication. In relation to waiting time, Azfar et al. asked specifically "bow many days would you be willing to wait to get a response from mobile teledermatology in exchange for the convenience of not having to travel so far or wait for a face-to-face consultation"? Interestingly, the majority (40\%) of participants answered 1-3 days compared with 19\% who wanted the answer on the same day. This is similar in some ways to the wait attribute results found in this study where the level "up to 1 day" was preferred over both "less than 4 hours" and "up to 3 days" (although, this only reached significance at the $10 \%$ level). It is uncertain why participants may prefer not to receive results straight away, but this may reflect peoples' assumptions about how long it will take for results to be processed. Future research should explore this question in more detail.

The use of a DCE methodology here is an advance on the existing literature which relied on the use of satisfaction rating scales, which don't allow for the relative importance of different aspects of the service to be directly compared [37]. This is important when designing how teledermoscopy might be incorporated into the current health system as it helps to inform decisions about who reviews the results, in what time frame and the acceptance of any out-ofpocket costs. The indicative welfare gain of AU $\$ 110$ to have teledermoscopy available as an additional screening option strongly suggests this screening mode could provide a societal welfare gain, even after considering the comparative costs of providing this service.

Nevertheless, given the limitations associated with the sample (discussed below), this estimate requires confirmation in larger representative samples before being implemented. The results however indicate that participants found the option of teledermoscopy valuable and would likely to take it up if it was available, all other things being equal. This estimate is also sensitive to the assumption that skin cancer clinics are staffed by GPs rather than dermatologists and the estimate drops to AU $\$ 52$ if this assumption is not met in practice. Given the relative shortage of dermatologists, we think the implementation of teledermoscopy is more feasible than skin cancer clinics being predominantly staffed by dermatologists, at least in the short to medium term.

In terms of limitations, this study used a small homogenous sample of participants at moderate to high risk of melanoma, all with experience using teledermoscopy in a previous component of this study [11]. As such, the generalizability of these results are limited. Nevertheless, the preferences elicited in this DCE are indicative of the likely preferences of a group of consumers who are at moderate to high risk of skin cancer and who are therefore a population of particular interest for targeted screening strategies. Further, due to the particular nature of the Australian health service, where GP visits are publicly funded and access to a dermatologist requires a GP 
referral, results cannot inform policy makers in other countries without further confirmation. However, this study outlines an approach to undertaking a similar study in a larger international sample, which would be advisable to inform implementation of teledermoscopy on a global scale.

Overall, we found that many of the aspects of skin cancer screening and skin cancer triage offered by teledermoscopy are valued by consumers at moderate to high risk of skin cancer, which is an important consideration for health service design. Teledermoscopy is an innovative technology which potentially offers at least equivalent health outcomes for consumers, delivered in a more acceptable way and may offer improved health service efficiency.

Conflict of interest: (see title page for anonymity of peer review process)

Acknowledgements: (see title page for anonymity of peer review process) 
Table 1: Attributes and levels used in the Discrete Choice Experiment (DCE)

\begin{tabular}{|c|c|c|}
\hline $\begin{array}{l}\text { Attributes (name used in } \\
\text { model) }\end{array}$ & Health service A or B +/- SSE & $\begin{array}{l}\text { Skin self- } \\
\text { examination (SSE) } \\
\text { only^ }^{\wedge}\end{array}$ \\
\hline Method of screening (type) & $\begin{array}{l}\text { Diagnosis using a phone camera } \\
\text { (type1) } \\
\text { Visit a skin cancer clinic (type2) } \\
\text { Visit your GP (base) }\end{array}$ & Checking yourself \\
\hline Time away from & More than 4 hours (time1) & The time it takes to \\
\hline home/office/usual activities & 3-4 hours (time2) & check your whole \\
\hline including travel (time) & 1-2 hours (base) & body \\
\hline $\begin{array}{l}\text { Chance of detection of melanoma } \\
\text { if one is present (chance) }\end{array}$ & $\begin{array}{l}\text { More than } 95 \% \text { (chance } 1) \\
85-95 \% \text { (chance } 2) \\
65-75 \% \text { (base) }\end{array}$ & $50 \%$ or less (constant) \\
\hline Wait time to get result (wait) & $\begin{array}{l}\text { Up to } 3 \text { days (wait1) } \\
\text { Up to } 1 \text { day (wait } 2 \text { ) } \\
\text { Less than } 4 \text { hours (base) }\end{array}$ & blank. \\
\hline Who reviews the result (who) & $\begin{array}{l}\text { GPt (1) (who) } \\
\text { Telederm dermatologist (base) }\end{array}$ & No-one \\
\hline $\begin{array}{l}\text { Number of non-cancerous moles } \\
\text { removed to find one melanoma } \\
\text { (benign) }\end{array}$ & $\begin{array}{l}3 \text { non-cancerous for one skin } \\
\text { cancer (benign1) } \\
5 \text { non-cancerous for one skin } \\
\text { cancer (benign2) } \\
10 \text { non-cancerous for one skin } \\
\text { cancer (base) }\end{array}$ & None \\
\hline Out of pocket cost* (cost) & continuous & No cost \\
\hline
\end{tabular}

* not including additional costs for biopsy or follow-up tests or treatment

+ constrained to be a GP who reviews the result if appearing with screening being performed at a skin cancer clinic or GP clinic

$\wedge$ The levels for the SSE were described in the survey text 
Table2: An example choice question

Question 1: Which routine skin check would you prefer from the following choices? Please tick your choice.

\begin{tabular}{|c|c|c|c|c|}
\hline & Health Service A & Health Service B & $\begin{array}{c}\text { Skin self- } \\
\text { examination }\end{array}$ & $\begin{array}{c}\text { I would } \\
\text { not }\end{array}$ \\
\hline Method of screening & $\begin{array}{c}\text { Visit a skin cancer } \\
\text { clinic }\end{array}$ & $\begin{array}{c}\text { Diagnosis using a phone } \\
\text { camera }\end{array}$ & & $\begin{array}{c}\text { perform } \\
\text { a }\end{array}$ \\
\hline $\begin{array}{l}\text { Time away from usual } \\
\text { activities }\end{array}$ & more than 4 hours & more than 4 hours & & $\begin{array}{l}\text { routine } \\
\text { skin }\end{array}$ \\
\hline $\begin{array}{l}\text { Chance of accurately } \\
\text { diagnosing a skin } \\
\text { lesion }\end{array}$ & $65-75 \%$ & $65-75 \%$ & & check \\
\hline $\begin{array}{l}\text { Wait time to get } \\
\text { result }\end{array}$ & up to 1 day & up to 3 days & & \\
\hline $\begin{array}{l}\text { Who reviews the } \\
\text { result }\end{array}$ & $\begin{array}{l}\text { GP performing the } \\
\text { service }\end{array}$ & A dermatologist & & \\
\hline $\begin{array}{l}\text { Number of non- } \\
\text { cancerous lesions } \\
\text { removed to detect } \\
\text { one skin cancer }\end{array}$ & $\begin{array}{l}10 \text { removed to detect } \\
\text { one skin cancer }\end{array}$ & $\begin{array}{l}3 \text { removed to detect } \\
\text { one skin cancer }\end{array}$ & & \\
\hline Out of pocket cost & $\$ 60$ & 0 & & \\
\hline
\end{tabular}


Table 3: Summary of participant characteristics

\begin{tabular}{|l|c|c|c|}
\hline Variable & Description & Proportion & Percentage \\
\hline Age & $50-54$ years & $19 / 35$ & 54 \\
\hline & $55-59$ years & $12 / 35$ & 34 \\
\hline Gender & $60-64$ years & $4 / 35$ & 11 \\
\hline Education & female & $19 / 35$ & 54 \\
\hline & university degree & $19 / 35$ & 54 \\
\hline & post-school certificate & $10 / 35$ & 29 \\
\hline Employment & high school or less & $6 / 35$ & 17 \\
\hline Income & employed full/part time & $26 / 35$ & 74 \\
\hline & $>\$ 60,000$ & $28 / 35$ & 80 \\
\hline Geographical location & $<\$ 60,000$ & $4 / 35$ & 11 \\
\hline & prefer not to say & $3 / 35$ & 9 \\
\hline & major city & $30 / 35$ & 86 \\
\hline Ever had skin cancer removed & regional centre & $4 / 35$ & 11 \\
\hline Diagnosis of skin cancer & not moles or warts & $1 / 35$ & 3 \\
\hline
\end{tabular}

* All 5 skin cancers detected in the previous teledermoscopy trial were basal cell or squamous cell carcinomas (BCCs or SCCs). No melanomas were detected in the trial [11]. 
Table 4: Result of mixed multinomial logit (MMNL) model

\begin{tabular}{|c|c|c|c|c|c|c|c|}
\hline \multirow[b]{2}{*}{ Attribute description } & \multirow[b]{2}{*}{ MMNL } & & \multirow[b]{2}{*}{ s.e. } & \multicolumn{2}{|c|}{$95 \% \mathrm{CI}$} & \multicolumn{2}{|c|}{ RP dist } \\
\hline & & & & lower & upper & s.d. & \\
\hline teledermoscopy screening & -0.046 & & 0.129 & -0.298 & 0.207 & & \\
\hline skin cancer clinic screening & 0.172 & & 0.137 & -0.096 & 0.440 & & \\
\hline base: GP screening & -0.127 & & & & & & \\
\hline time away from usual activities $>4$ hours & -0.233 & $*$ & 0.125 & -0.478 & 0.011 & & \\
\hline time away from usual activities 3-4 hours & -0.144 & & 0.149 & -0.436 & 0.148 & & \\
\hline base: $1-2$ hours & 0.377 & & & & & & \\
\hline$>95 \%$ chance of skin cancer detection & 0.865 & $* * *$ & 0.180 & 0.513 & 1.218 & 0.628 & $* * *$ \\
\hline $85-95 \%$ chance of skin cancer detection & 0.202 & $*$ & 0.119 & -0.031 & 0.436 & & \\
\hline base: $65-75 \%$ chance of skin cancer detection & -1.068 & & & & & & \\
\hline wait time to get results up to 3 days & -0.069 & & 0.132 & -0.328 & 0.190 & & \\
\hline wait time to get results up to 1 day & 0.258 & $*$ & 0.132 & -0.001 & 0.516 & & \\
\hline base: wait time to get results $<4$ hours & -0.189 & & & & & & \\
\hline Result reviewed by GP & -0.458 & $* * *$ & 0.114 & -0.681 & -0.235 & 0.344 & $* * *$ \\
\hline base: Result reviewed by dermatologist & 0.458 & & & & & & \\
\hline 3 non-cancerous moles removed for 1 skin cancer & 0.532 & $* * *$ & 0.164 & 0.210 & 0.855 & 0.514 & $* * *$ \\
\hline 5 non-cancerous moles removed for 1 skin cancer & -0.061 & & 0.123 & -0.303 & 0.180 & & \\
\hline base: 10 non-cancerous moles removed for 1 skin cancer & -0.471 & & & & & & \\
\hline Cost (continuous) & -0.010 & $* * *$ & 0.004 & -0.017 & -0.003 & & \\
\hline Skin self-examination & -1.987 & $* * *$ & 0.217 & -2.412 & -1.562 & & \\
\hline McFadden Pseudo R-squared & 0.328 & & & & & & \\
\hline
\end{tabular}

$* * *, * *, *$ indicates significance at the $1 \%, 5 \%$ and $10 \%$ level respectively.

Abbreviations: $\mathrm{MMNL}=$ mixed multinomial logit; $\mathrm{CI}=$ confidence interval; $\mathrm{RP}=$ random parameter; dist $=$ distribution; s.e. $=$ standard error; s.d.= standard deviation; 
Table 5: Willingness to Pay for teledermoscopy to be available as an additional alternative

\begin{tabular}{|l|c|c|c|c|}
\hline & \multicolumn{3}{|c|}{ Probability of uptake } \\
\hline & SSE & $\begin{array}{c}\text { Skin cancer } \\
\text { clinic }\end{array}$ & GP & teledermoscopy \\
\hline $\begin{array}{l}\text { Three available } \\
\text { alternatives }\end{array}$ & 0.037 & 0.553 & 0.410 & NA \\
\hline $\begin{array}{l}\text { Four available } \\
\text { alternatives }\end{array}$ & 0.012 & 0.183 & 0.136 & 0.668 \\
\hline $\begin{array}{l}\text { Willingness-to-pay for the situation where teledermoscopy is available } \\
\text { additional to existing three options: }\end{array}$ & $\$ 110$ \\
\hline
\end{tabular}

Abbreviations: SSE = skin self-examination; GP = general practitioner

Notes: (1) The 'initial state of the world' includes 3 available alternatives: SSE, skin cancer clinic screening \& GP screening. The 'state of world' after policy change includes four available alternatives: SSE, skin cancer clinic screening, GP screening \& teledermoscopy.

(2) The estimates for probability of uptake assume differences in three attributes: (i) the type attribute; (i) who reviews the results; and (ii) the benign attribute. The level of the benign attribute for the teledermoscopy alternative is set to the ' 3 non-cancerous moles removed for 1 skin cancer' level, whereas this is ' 5 non-cancerous moles' for GP and skin cancer clinic alternatives. Dermatologist review is assumed for the teledermoscopy alternative, GP review is assumed for the skin cancer and GP clinic alternatives. The best levels for all other attributes are chosen and held constant across the GP, skin cancer clinic and teledermoscopy alternatives.

(3) The Small and Rosen formula used to calculate compensating variation is shown in Appendix 2. 


\section{Appendix 1: Theoretical framework for analysis}

The DCE data were analysed in a conventional random utility theoretical framework using regression models, in which the attribute levels (independent variables) were used to explain screening choice (dependent variable). The most-widely used choice model is the multinomial logit (MNL) model which estimates the average contribution to utility, or satisfaction, across the sample of each attribute level compared to its base level. The utility function may be expressed as:

\section{Equation 1: $\quad U_{n j}-V_{m i}+a_{m i}$}

where $\mathrm{U}$ is the utility the $n^{\text {th }}$ consumer derives from alternative $j$; $\mathrm{V}$ is the observed component of utility (comprising of a linear combination of parameters $(\beta)$ and attribute levels $(x k)$, as specified in Equation 2), and $\varepsilon$ is the unobserved component (idiosyncratic error term).

\section{Equation 2:}

$$
V_{n j}=\beta_{n k k} x_{n j k}
$$

One of the limitations of the MNL model is that it imposes quite restrictive assumptions, including the homogeneity of preferences and independence of choices even over repeated choice questions, which are likely to be violated [32]. For this reason, the mixed logit (MMNL) model is often preferred to the MNL as all of the restrictive assumptions of the MNL may be relaxed $[32,33]$. In a MMNL model, one or more of the parameters in the model are treated as random, as represented by:

\section{Equation 3: $\quad \beta_{n k}=\beta_{k}+n_{k} z_{m s k}$}

where $\tilde{\beta}_{k}$ is the marginal mean utility in the sample; $n_{k}$ is the deviation of the mean marginal utility for respondent $n$ for attribute $k$, which is part of alternative $j$ in choice question $s$; and $\boldsymbol{z}_{\mathrm{m} / \mathrm{k}}$ represents an underlying distribution (such as normal, triangular or log-normal). 
Appendix 2: The Small and Rosen formula for compensating variation.

$C V=\frac{1}{\gamma}\left[\operatorname{In} \sum_{f=1}^{L} e^{V_{f}^{\theta}}-\operatorname{In} \sum_{i=1}^{L} e^{V_{l}^{H}}\right]$

where: $\mathrm{CV}=$ compensating variation - a measure of how much money needs to be given or taken from a consumer after a price or quality change to leave them at their initial level of utility; $\gamma=$ the marginal utility of income (approximated here by the negative coefficient of the cost attribute); $V_{f}^{0}$ and $V_{f}^{1}$ are the values of the indirect utility functions for each choice option $j$ before and after the quality (attribute level) change; and $J$ is the number of options in the choice set.

From: Small, KA \& Rosen, HS (1981). Applied welfare economics with discrete choice models. Econometrica; 49:105-130. 


\section{References}

1. Cancer Council of Queensland, Skin Cancer - Fact Sheet. 2011 [cited 24/02/2015]; Available from: http://www.cancerqld.org.au/icms_docs/61890_Skin_cancer_fact_sheet.pdf.

2. Queensland Cancer Registry, Cancer in Queensland: Incidence, Mortality, Survival and Prevelence 19822011, Queensland Health and Cancer Council, 2013: Brisbane, Australia.

3. Non-melanoma Skin Cancer Working Group, The 2002 national non-melanoma cancer survey, 2003: Canberra, Australia.

4. Cancer Council of Australia, About skin cancer. 2015 [cited 16/03/2015]; Available from: http://www.cancer.org.au/preventing-cancer/sun-protection/about-skin-cancer.html.

5. Whiteman, D.C., P.D. Baade, and C.M. Olsen, More People Die from Thin Melanomas (less than $1 \mathrm{~mm}$ ) than from Thick. Melanomas (greater than 4mm) in Queensland, Australia. J Invest Dermatol, 2014.

6. Aitken, J., et al., Prevalence of Whole-Body Skin Self-Examination in a Population at High Risk. for Skin Cancer (Australia). Cancer Causes \& Control, 2004. 15(5): p. 453-463.

7. Cancer Council of Australia, Checking for signs of skin cancer. 2015 [cited 25/03/2015]; Available from: http://www.cancer.org.au/preventing-cancer/sun-protection/check-for-signs-of-skincancer.html.

8. Wilkinson, D., D.A. Askew, and A. Dixon, Skin cancer clinics in Australia: workload profile and performance indicators from an analysis of billing data. Medical Journal of Australia, 2006. 184(4): p. 162 4.

9. $\quad$ Massone, C., et al., Melanoma Screening with Cellular Phones. PLoS One, 2007. 2(5).

10. Kroemer, S., et al., Mobile teledermatology for skin tumour screening: diagnostic accuracy of clinical and dermoscopic image tele-evaluation using cellular phones. British Journal of Dermatology, 2011. 164(5): p. 973-979.

11. Manahan, M.N., et al., A pilot trial of mobile, patient-performed teledermoscopy. British Journal of Dermatology, 2015: p. n/a-n/a.

12. Tan, E., et al., Successful triage of patients referred to a skin lesion clinic using teledermoscopy (IMAGE IT trial). British Journal of Dermatology, 2010. 162(4): p. 803-811.

13. Massone, C., et al., Teledermatology for skin cancer prevention: an experience on 690 Austrian patients. Journal of the European Academy of Dermatology and Venereology, 2014. 28(8): p. 1103-1108.

14. Wu, X., et al., Feasibility and efficacy of patient-initiated mobile teledermoscopy for short-term monitoring of clinically atypical nevi. JAMA Dermatology, 2015.

15. Piccolo, D., et al., Dermoscopic diagnosis by a trained clinician vs. a clinician with minimal dermoscopy training vs. computer-aided diagnosis of 341 pigmented skin lesions: a comparative study. British Journal of Dermatology, 2002. 147(3): p. 481-486.

16. Azfar, R.S., et al., HIV-positive patients in Botswana state that mobile teledermatology is an acceptable method for receiving dermatology care. Journal of Telemedicine and Telecare, 2011. 17(6): p. 338-340.

17. Collins, K., S. Walters, and I. Bowns, Patient satisfaction with teledermatology: quantitative and qualitative results from a randomized controlled trial. Journal of Telemedicine and Telecare, 2004. 10(1): p. 29-33.

18. Demiris, G., S. Speedie, and L. Hicks, Assessment of Patients' Acceptance of and Satisfaction with Teledermatology. Journal of Medical Systems, 2004. 28(6): p. 575-579.

19. Lim, D., A.M.M. Oakley, and M. Rademaker, Better, sooner, more convenient: A successful teledermoscopy service. Australasian Journal of Dermatology, 2012. 53(1): p. 22-25.

20. Tyagi, A., K. Miller, and M. Cockburn, e-Health Tools for Targeting and Improving Melanoma Screening: A Review. Journal of Skin Cancer, 2012. 2012: p. 8.

21. Weinstock, M.A., F.Q. Nguyen, and P.M. Risica, Patient and referring provider satisfaction with teledermatology. Journal of the American Academy of Dermatology, 2002. 47(1): p. 68-72.

22. Whited, J.D., et al., Patient and Clinician Satisfaction with a Store-and-Forward Teledermatology Consult System. Telemedicine Journal and e-Health, 2004. 10(4): p. 422-431.

23. de Bekker-Grob, E.W., M. Ryan, and K. Gerard, Discrete choice experiments in health economics: a review of the literature. Health Economics, 2012. 21(2): p. 145-172.

24. Lancsar, E. and J. Louviere, Conducting discrete choice experiments to inform bealthcare decision making: a user's guide. PharmacoEconomics, 2008. 26: p. 661+.

25. Ryan, M., Discrete choice experiments in health care. Vol. 328. 2004. 360-361.

26. Bridges, J.F.P., et al., Conjoint Analysis Applications in Health-a Checklist: A Report of the ISPOR Good Research Practices for Conjoint Analysis Task. Force. Value in Health, 2011. 14(4): p. 403-413. 
27. Warshaw, E.M., et al., Teledermatology for diagnosis and management of skin conditions: A systematic review. Journal of the American Academy of Dermatology, 2011. 64(4): p. 759-772.e21.

28. Whited, J.D., Teledermatology research review. International Journal of Dermatology, 2006. 45(3): p. 220-229.

29. Rose, J.M., et al., Designing efficient stated choice experiments in the presence of reference alternatives. Transportation Research Part B: Methodological, 2008. 42(4): p. 395-406.

30. Rose, J.M. and M.C.J. Bliemer, Constructing Efficient Stated Choice Experimental Designs. Transport Reviews, 2009. 29(5): p. 587-617.

31. Levin, I.P. and G.J. Gaeth, How Consumers are Affected by the Framing of Attribute Information Before and After Consuming the Product. Journal of Consumer Research, 1988. 15(3): p. 374-378.

32. Train, K., Discrete choice methods with simulation. 2009, New York: Cambridge University Press.

33. Hensher, D.A., J.M. Rose, and W.H. Greene, Applied choice analysis. 2005, New York: Cambridge University Press.

34. Lancsar, E. and E. Savage, Deriving welfare measures from discrete choice experiments: inconsistency between current methods and random utility and welfare theory. Health Economics, 2004. 13(9): p. 901-907.

35. Ryan, M., Deriving welfare measures in discrete choice experiments: a comment to Lancsar and Savage (1). Health Economics, 2004. 13(9): p. 909-912.

36. Small, K. and H. Rosen, Applied welfare economics with discrete chocie models. Econometrica, 1981. 49: p. 105-130.

37. Hensher, D.A., Stated preference analysis of travel choices: the state of practice. Transportation (19861998), 1994. 21(2): p. 107. 